\title{
COMPARISON OF VARIOUS MODELS IMPLEMENTED USING LINEAR AND NON-LINEAR SYSTEM IDENTIFICATION METHODS FOR CEMENT GRINDING PROCESS USING VERTICAL ROLLER MILL
}

\author{
VIJAYA BHASKAR. B ${ }^{1} \&$ JAYALALITHA $S^{2}$ \\ ${ }^{l}$ Assistant Professor, EIE Department, SASTRA University, Thanjavur, Tamil Nadu, India \\ ${ }^{2}$ Associate Dean, EIE Department, SASTRA University, Thanjavur, Tamil Nadu, India
}

Vertical Roller Mill (VRM) has been promptly employed and extensively used for clinker and cement grinding for past 35 years, because of its higher efficiency and low power consumption. VRM is a highly nonlinear, time delay and composite industrial process with several process loops having a robust coupling between them. Time series data is collected from a real time cement plant with grinding capacity of 170-300 tph for system identification purpose. The collected industrial data is normalized and preprocessed using a moving average window filter to remove odd samples; trends and means are removed for outlier elimination and missing values, then the data is divided into an estimation data and a validation data. In this paper linear and nonlinear system identification methods are applied to identify the VRM model. Finally, this paper proposes an accurate VRM model for industrial requirement to adopt modern control techniques like Model Predictive Control (MPC) and Adaptive Control (AC) for cement grinding in order to improve its automatic control level.

KEYWORDS: Vertical Roller Mill, System Identification, Hammerstein Model, Polynomial Model \& Auto Regressive Models
\end{abstract}

Received: Jan 10, 2018; Accepted: Jan 31, 2018; Published: Feb 26, 2018; Paper Id.: IJMPERDAPR201815

\section{INTRODUCTION}

VRMs have evolved through the years and are currently being used at various locations worldwide for grinding slag, OPC grade 43 and 53, PPC and PSC cement. Clinker and cement grinding machines are the maximum energy consumption units in the cement manufacturing process. Figure 1. Shows the various parts of the VRM used for cement grinding. Figure 2. Shows the snapshot of the real time VRM used for cement grinding. The working principle of VRM involves start, steady state stop processes. The important processes present in VRM are grinding, drying and separation. 


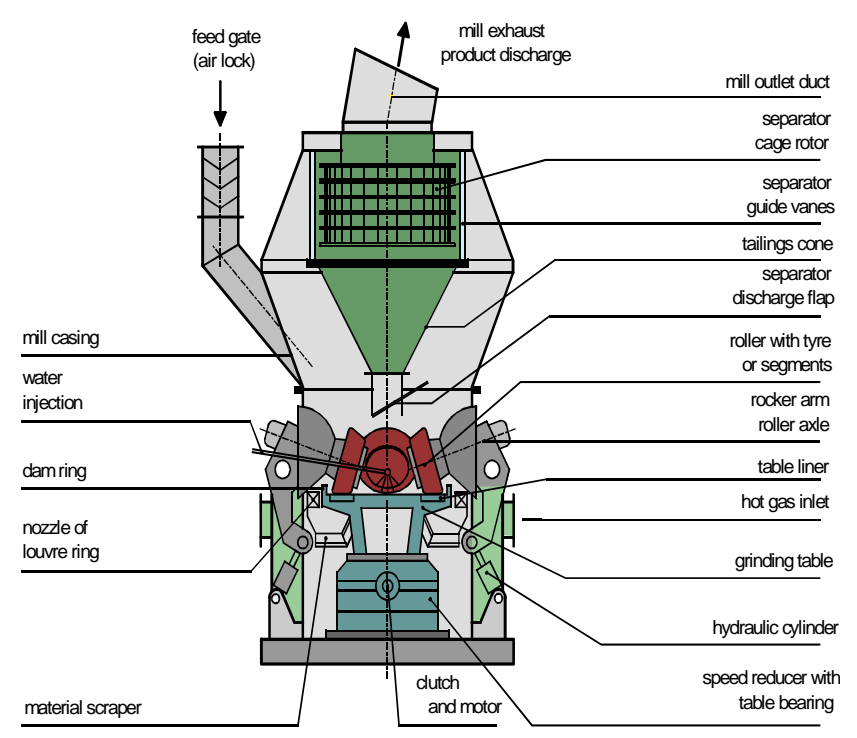

Figure 1: Parts of Vertical Roller Mill Adopted from https://www.scribd.com/doc/97749158/ Vrm-Presentation

A collective appliance of pressure frame, hydraulic tensioning system and pull rod is used to force the rollers against the grinding bed. From the air stream the completed product is detached and conveyed to the cement silos [1], [2] and [6].

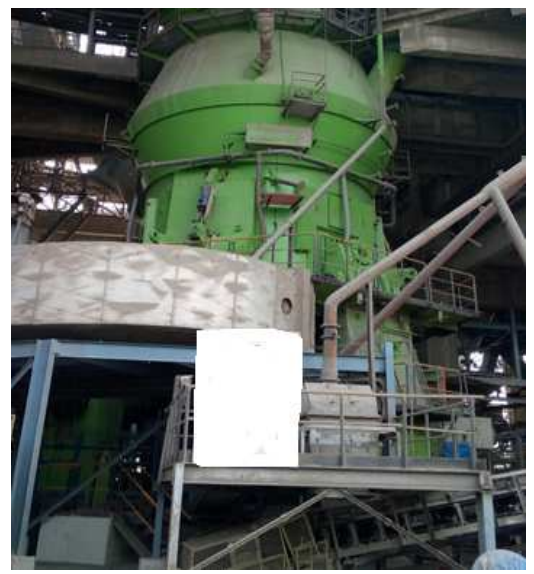

Figure 2: Photo of the Real Time VRM Used in Cement Plant

\section{MODEL IDENTIFICATION}

The model identification is the development and investigation of the models in accordance with the input-output data. Models are built from basic physical laws and other well recognized relationships. The flow chart of the system identification loop using input-output data is shown in Figure 3. The extensively used linear models are transfer function models, Linear Auto regressive (LARX) models, State space models and polynomial models. The time series input-output data is the basis for system identification irrespective of the model structure [3]. The generalized model structure and its predictor are represented and expressed as (1) and (2).

$$
A(p)^{*} y(t)=p^{-n_{k} y(t)} \frac{\bar{B}(p)}{F(p)} * u(t)+\frac{C(p)}{D(p)} * e(t)
$$




$$
\widehat{y}(t \mid \theta)=\frac{D(p) B(p)}{C(p) F(p)} u(t)+\left[1-\frac{D(p) A(p)}{C(p)}\right] y(t)
$$

Where, $u(t)$ - cause, $y(t)$ - response, $e(t)$ - disturbance, $A(p), B(p), C(p), D(p)$, and $F(p)$ - matrices with unknown parameters $p$.

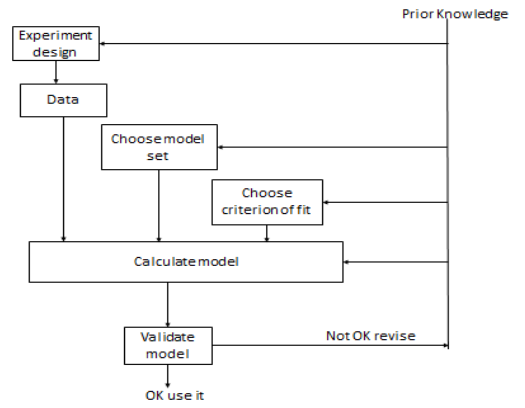

Figure 3: Flow Chart of System Identification

\section{LINEAR ESTIMATION METHODS}

The mathematical model commonly expresses the dependencies of various process variables. Transfer function model is the most widely used methods for modelling dynamic linear time-invariant systems. The transfer function models are discussed in [4], [5] and [7].

\subsection{Transfer Function Models}

Transfer function based mathematical models are more appropriate for the time domain, frequency-domain and stability analysis of the systems. Continuous and a discrete time systems are expressed as (3) and (4).

$$
\begin{aligned}
& y(t)=G(s) * u(t)+e(t) \\
& y(k)=G(z) * u(k)+e(k)
\end{aligned}
$$

Where, $G(s)$ and $G(z)$ - transfer function between the cause and the effect.

The transfer function models for single input- single output continuous time and discrete time systems are expresses as (5) and (6).

$$
\begin{gathered}
G(s)=\frac{b_{0}+b_{1} s+\cdots+b_{m-1} s^{m-1}+b_{m} s^{m}}{a_{0}+a_{1} s+\cdots a_{n-1} s^{n-1}+a_{n} s^{n}} \\
G(z)=\frac{b_{0}+b_{1} z+\cdots+b_{m-1} z^{m-1}+b_{m} z^{m}}{a_{0}+a_{1} z+\cdots a_{n-1} z^{n-1}+a_{n} z^{n}}
\end{gathered}
$$

Where, $b_{m}$ and $a_{n^{-}}$coefficients of the numerator polynomial function, $m$ and $n$ - order of the numerator and denominator.

The equations for the continuous and discrete transfer function of multi input single output systems are 


$$
\begin{aligned}
& y_{i}=\sum_{j=1}^{n} G_{i j}(s) u_{j} \\
& y_{i}=\sum_{j=1}^{n} G_{i j}(z) u_{j}
\end{aligned}
$$

Where, $G_{i j^{-}}$transfer functions between the cause and the effect, $I$ and $j$ - number of inputs and outputs.

The algorithmic steps to construct transfer function model is as follows

Step1: Apply Prediction Error Method to calculate discrete model

Step2: Convert discrete model to a continuous model by applying Zero-Order-Hold

Step3: Optimize continuous model by using Gauss-Newton method

\subsection{Polynomial Models}

Polynomial models separately define the stochastic and deterministic parts of a system where as transfer function models define only the deterministic part. The polynomial models are discussed in [8]. Different polynomial models widely used for system identification include ARX models, Auto regressive Moving Average models, Instrument variable models, Box-Jenkins Model and Output error model.

\subsubsection{ARX Modelling}

Figure 4 shows the ARX model block diagram. The linear difference equation represents the input-output relationship as

$$
\begin{aligned}
& y(t)+a_{1} * y(t-1)+\cdots \cdot+a_{n_{a}} * y\left(t-n_{a}\right)= \\
& b_{1} * u(t-1)+\cdots \cdots+b_{n_{b}} * u\left(t-n_{b}\right)+e(t)
\end{aligned}
$$

Where, $\quad \theta=\left[\begin{array}{lllll}a_{1} & a_{2} \cdots & a_{n} & b_{1} \cdots b_{n_{b}}\end{array}\right]^{T} \quad$ is the adjustable parameter, $A(p)=1+a_{1} * p^{-1}+\cdots+a_{n} * p^{-n_{a}}$ and $B(p)=b * p^{-1}+\cdots+b_{n_{b}} * p^{-n_{b}}$ then $G(p, \theta)=\frac{B(p)}{A(p)}$ and

$$
H(p, \theta)=\frac{1}{A(p)}
$$

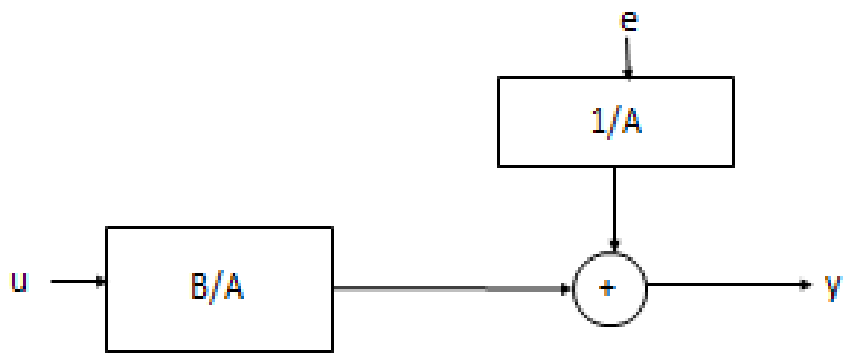

Figure 4: The ARX Model Structure 


\subsubsection{Instrument Variable (IV) Modelling}

In this modeling, variance and Mean Square Error (MSE) depend on the choice of instruments. If the noise and instruments are correlated or model order selection is wrong or filter dynamics cancels plant dynamics then consistency is lost. The IV model is shown in Figure 5.

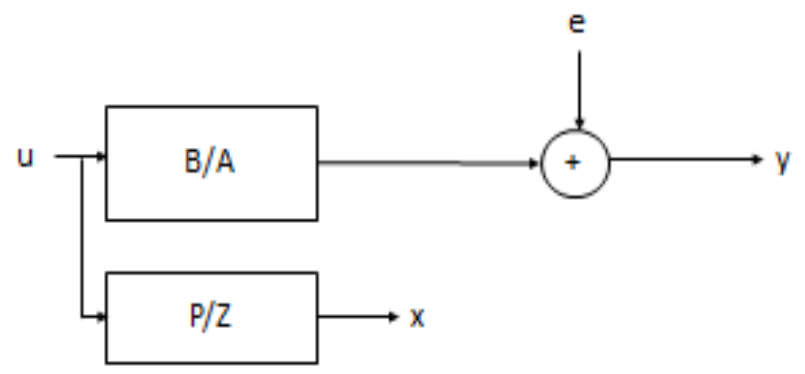

Figure 5: Model Structure for IV Method

\subsubsection{ARMAX Modelling}

In ARX model the properties of the noise term is not more adequate. In ARMAX model, moving average of white noise relates the noise model for more flexibility. The model equations are

$$
\begin{aligned}
& y(t)+a_{1} * y(t-1)+\cdots \cdots+a_{n_{a}} * y\left(t-n_{a}\right)= \\
& b_{1} * u(t-1)+\cdots+b_{n_{b}} * u\left(t-n_{b}\right)+e(t)+ \\
& c_{1} * e(t-1)+\cdots+c_{n_{c}} * e\left(t-n_{c}\right)
\end{aligned}
$$

By assuming $C(p)=1+c_{1} p^{-1}+\cdots+c_{n c} p^{-n c}$

Equation (11) is written as

$A(p) * y(t)=B(p) * u(t)+C(p) * e(t)$ and corresponds to

$$
G(p, \theta)=\frac{B(p)}{A(p)} \text { and } H(p, \theta)=\frac{C(p)}{A(p)}
$$

The ARMAX model structure is shown in Figure 6

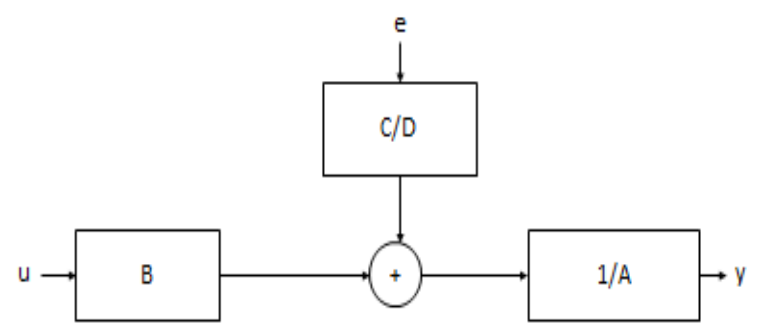

Figure 6: The Equation Error Model Family Model Structure

\subsubsection{Output Error (OE) Modelling}

In $\mathrm{OE}$ model structure the $G$ and $H$ transfer function denominators have the term $A$ as a mutual factor. Figure 7 
shows the structure of OE model. Let the undisturbed output $w$, then the model equations are as follows

$$
\begin{aligned}
& w(t)+f_{1} * w(t-1)+\cdots+f_{n_{f}} * w\left(t-n_{f}\right)= \\
& b_{1} * u(t-1)+\cdots+b_{n_{b}} * u\left(t-n_{b}\right) \\
& y(t)=w(t)+e(t) \\
& \text { For, } F(q)=1+f_{1} q^{-1}+\cdots+f_{n_{f}} q^{-n_{f}}, \text { the model and the parameter vectors are } \\
& y(t)=\frac{B(p)}{F(p)} * u(t)+e(t) \\
& \qquad\left.\begin{array}{lll}
b_{1} & b_{2}
\end{array} \quad b_{n_{b}} \quad f_{1} \quad f_{2} \cdots f_{n_{f}}\right|^{T}=\theta
\end{aligned}
$$

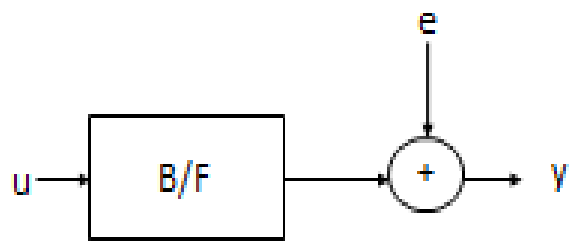

\section{Figure 7: The Output Error Model Block Diagram}

\subsubsection{Box-Jenkins Model Structure}

By describing the OE model as ARMA model gives

$$
y(t)=\frac{B(p)}{F(p)} * u(t)+\frac{C(p)}{D(p)} * e(t)
$$

The $G$ and $H$ transfer functions are parameterized as a rational functions independently. Figure 8 shows the NJ model structure.

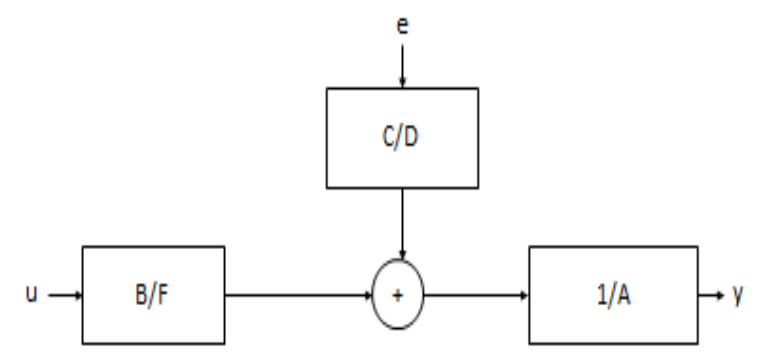

Figure 8: The BJ Model Structure

\subsection{State Space Modelling}

The relationship between the cause, response and noise is written as difference or differential equation in state space modelling by using an auxiliary state vector $x(t)$. The model equation is as follows 


$$
\dot{x}(t)=A(\theta) * x(t)+B(\theta) * u(t)
$$

Where, $A$ and $B$ - nxn and nxm matrices.

Let $m(t)$ be the ideal measurements obtained, $m(t)=H^{*} x(t)$ using $q$ for the differentiation operator the model equations is as follows,

$$
\left[q^{*} I-A(\theta)\right]^{*} x(t)=B(\theta) * u(t)
$$

The transfer operator from $u$ to $m$ is $m(t)=G_{c}(q, \theta) * u(t)$

$$
G_{c}(q, \theta)=H *[q * I-A(\theta)]^{-1} * B(\theta)
$$

\section{NONLINEAR MODELLING}

A non-linear relationship between the input and output sequences evidently provides superior possibilities to describe a system. The different input and output nonlinearities are wavelet network, piecewise linear function, saturation, sigmoid network, one-dimensional polynomial, dead zone, or a custom network. Nonlinear models are mainly of Nonlinear ARX models and Hammerstein-Wiener models.

\subsection{Nonlinear ARX (NARX) Modelling}

NARX model comprises of nonlinearity estimator and model regressors. Both linear and nonlinear functions are included in nonlinear estimator that acts on the model regressors to provide the model output [9]. Figure 9 shows the nonlinear ARX model.

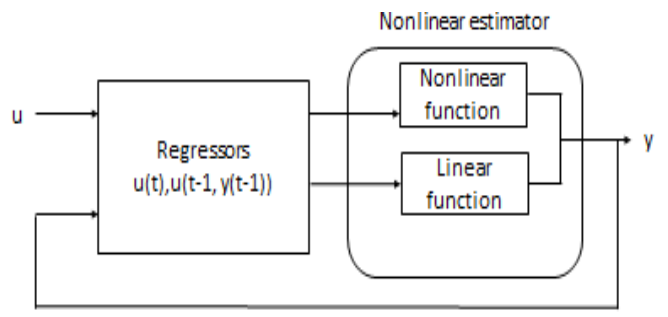

Figure 9: NARX Model Structure

The algorithmic steps of NARX modelling are as follows

Step 1: By using past output data, present and past input values calculate the regressor value.

Step 2: By using the nonlinearity estimator map the regressors to the model output.

\subsection{Hammerstein Modelling}

Hammerstein-Wiener models uses linear block in series with static nonlinear block to describe the dynamic systems [10], [11] and [12]. Figure 10 shows basic model of a Hammerstein-Wiener model. 


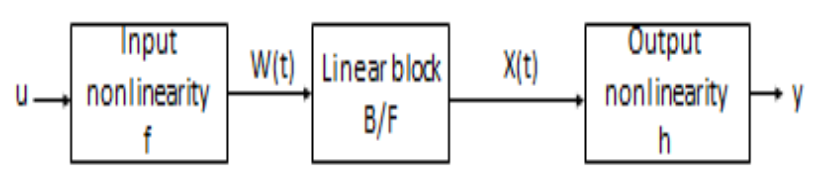

Figure 10: Model Structure of Hammerstein-Wiener

The algorithmic steps used in this modelling are as follows

Step 1: By using input data calculate $w(t)=f(u(t))$

Step 2: Using initial conditions $x(t)=(B / F)^{*} w(t)$ and $w(t)$ calculate the output of the linear block and configure it.

Step 3: By transforming the response of $x(t)$ calculate model output using nonlinear function $h$ as $y(t)=h(x(t))$ and configure it.

In most of the estimation methods Prediction Error Minimization (PEM) method is used for error minimization purpose. The algorithmic steps of PEM are as follows

Step1: Choose a model structure of the form

$$
y(t)=B\left(p^{-1} ; \theta\right) * u(t)+A\left(p^{-1} ; \theta\right) * e(t), E\left[e(t) e(t)^{*}\right]=\wedge(\theta)
$$

Step2: Choose a predictor of the form

$$
\widehat{y}(t \mid t-1 ; \theta)=L\left(p^{-1} ; \theta\right) * y(t)+M\left(p^{-1} ; \theta\right) * u(t)
$$

Step3: Select a criterion function $f(R(\theta))$

Step4: Determine $\widehat{\theta}$ that minimizes the loss function $f$

\section{MATHEMATICAL MODELLING OF VRM}

The difficulty to observe internal phenomena and the complexity of the VRM system do not allow a thorough knowledge process. Number of modelling methods are attempted to overcome these difficulties have been carried out in the last decades. The prediction of some key parameters in the operational process of VRM is very important for its safety and reliability. Because it works too long in poor conditions, its key operating parameters are nonlinear and time-varying and the traditional prediction methods are difficult to achieve high accuracy [13] and [14]. A different process parameters available VRM process is inlet feed, inlet pressure, outlet pressure, mill inlet temperature, mill outlet temperature, hot air supply, water injection, Separator speed, mill vibrations, grinding table speed, bed thickness and power consumption of the mill. The real time VRM process consists of three main and three supporting rollers. The data collected VRM mill specifications are:

(i) OPC: $210 \mathrm{tph}, 26.5 \mathrm{Kwh} / \mathrm{MT}$ cement

(ii) PPC: 300tph, $19.8 \mathrm{Kwh} / \mathrm{MT}$ cement

(iii) PSC: $170 t p h, 32.0 \mathrm{Kwh} / \mathrm{MT}$ cement

(iv) Cement grinding specific power: $24.5 \mathrm{Kwh}$ 
(v) Cement mill MTBF: $125 \mathrm{Hrs}$

(vi) Fly ash absorption: $34.2 \%$

(vii) Slag absorption: $58 \%$

From the industry expert knowledge, the selected input parameters are input feed (TPH), water injection pump speed (RPM) and output parameters are mill differential pressure (mbar), mill vibrations ( $\mathrm{mm} / \mathrm{sec})$, mill bed thickness $(\mathrm{mm})$ and mill power consumption (Kwh) for system identification. Figure 11 shows the real time plant data used for system identification after pre-processing.

\section{RESULTS}

From Table 1 the best fit model for the process variables differential pressure and mill vibrations is given by discrete time state space model; for the process variables mill bed thickness and mill power consumption the best fit is given by the method ARMAX. The best fit models are shown in Table 2 .
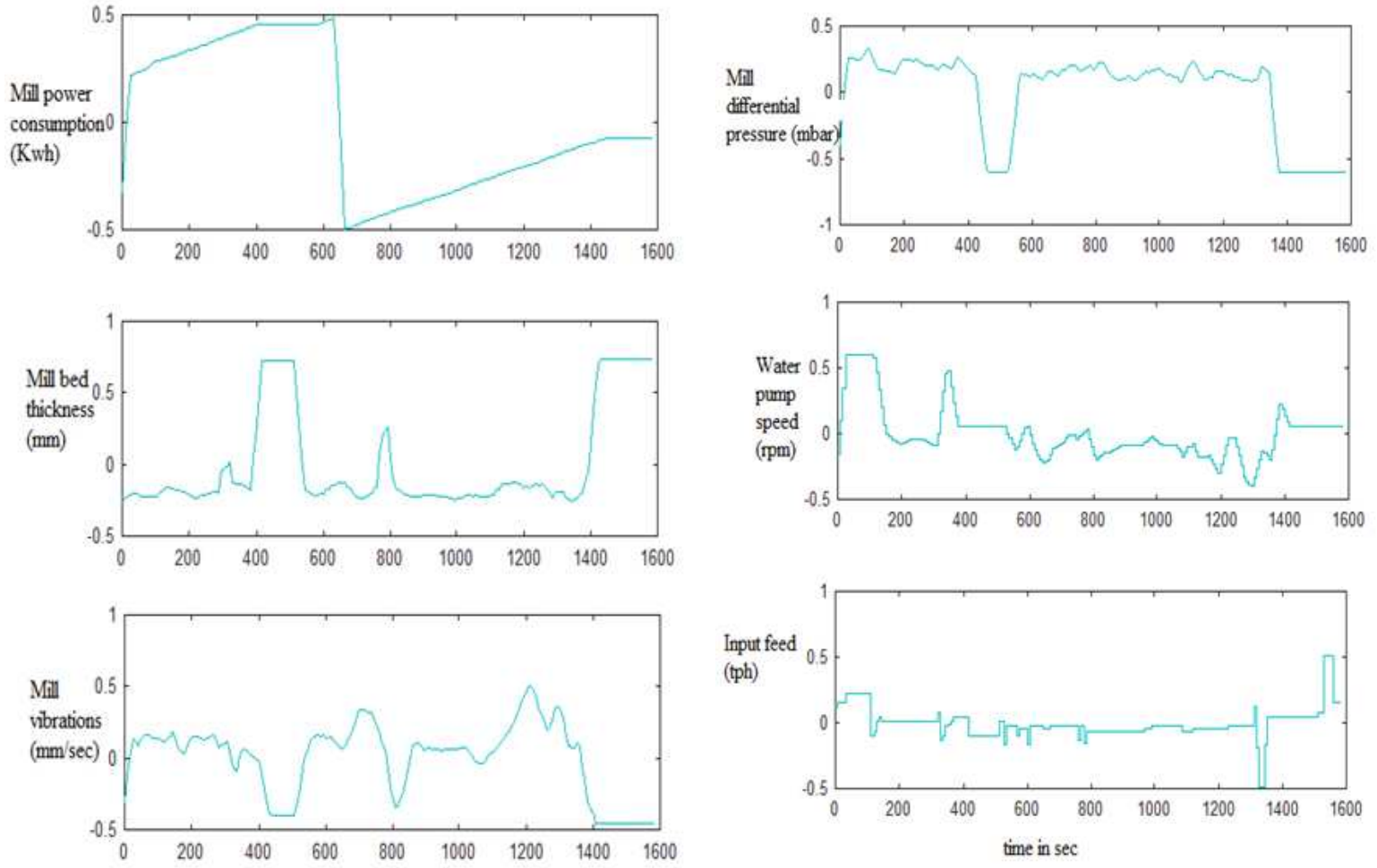

Figure 11: Real Time Pre-Processed Data For System Identification 
Table 1: Comparison of Various System Identification Methods Applied for VRM Modelling

\begin{tabular}{|l|l|c|c|c|c|c|c|}
\hline $\begin{array}{c}\text { Modelling } \\
\text { Method }\end{array}$ & Model Type & $\begin{array}{c}\text { Differential } \\
\text { Pressure } \\
(\mathbf{m b a r}) \\
\text { \% Fit }\end{array}$ & $\begin{array}{c}\text { Mill } \\
\text { Vibrations } \\
(\mathbf{m m} / \text { Sec) } \\
\text { \% Fit }\end{array}$ & $\begin{array}{c}\text { Mean } \\
\text { Square } \\
\text { Error }\end{array}$ & $\begin{array}{c}\text { Mill Bed } \\
\text { Thickness } \\
(\mathbf{m m}) \\
\text { \% Fit }\end{array}$ & $\begin{array}{c}\text { Mill Power } \\
\text { Consumption } \\
\text { (Kwh) } \\
\text { \% Fit }\end{array}$ & $\begin{array}{c}\text { Mean } \\
\text { Square } \\
\text { Enror }\end{array}$ \\
\hline \multirow{3}{*}{ State space model } & Continuous time & 92.02 & 89.1 & 0.00135 & 86.95 & 91.67 & 0.00291 \\
\cline { 2 - 8 } & Discrete time & $\mathbf{9 2 . 5 8}$ & $\mathbf{8 9 . 5 1}$ & 0.001213 & 87.29 & 89.79 & 0.003178 \\
\hline \multirow{5}{*}{ Polynomial models } & LARX & 89.72 & 88.04 & 0.001906 & 87.03 & 92.36 & 0.002761 \\
\cline { 2 - 8 } & IV & 46.91 & 35.5 & 0.1413 & 78.4 & 79.6 & 0.01044 \\
\cline { 2 - 8 } & ARMAX & 89.44 & 88.64 & 0.001873 & 88.53 & $\mathbf{9 2 . 5 2}$ & 0.002272 \\
\cline { 2 - 8 } & OE & 51.98 & 6.546 & 0.07648 & 28.91 & 50.33 & 0.09075 \\
\cline { 2 - 8 } & BJ & 89.83 & 81.27 & 0.003174 & 87.59 & 92.14 & 0.002621 \\
\hline \multirow{2}{*}{ Non- linear models } & NARX & 89.61 & 87.94 & 0.001948 & 88.14 & 92.45 & 0.002399 \\
\cline { 2 - 8 } & Hammerstein-Wiener & 21.73 & 21.84 & 0.09685 & 24.27 & 72.54 & 0.0806 \\
\hline \multirow{2}{*}{ Transfer function models } & Continuous time & 45.13 & 60.34 & 0.05375 & 60.81 & 71.59 & 0.02821 \\
\cline { 2 - 8 } & Discrete time & 32.45 & 42.16 & 0.06465 & 30 & 24.48 & 0.1243 \\
\hline
\end{tabular}

Table 2: Best Fit Model Results for VRM Process

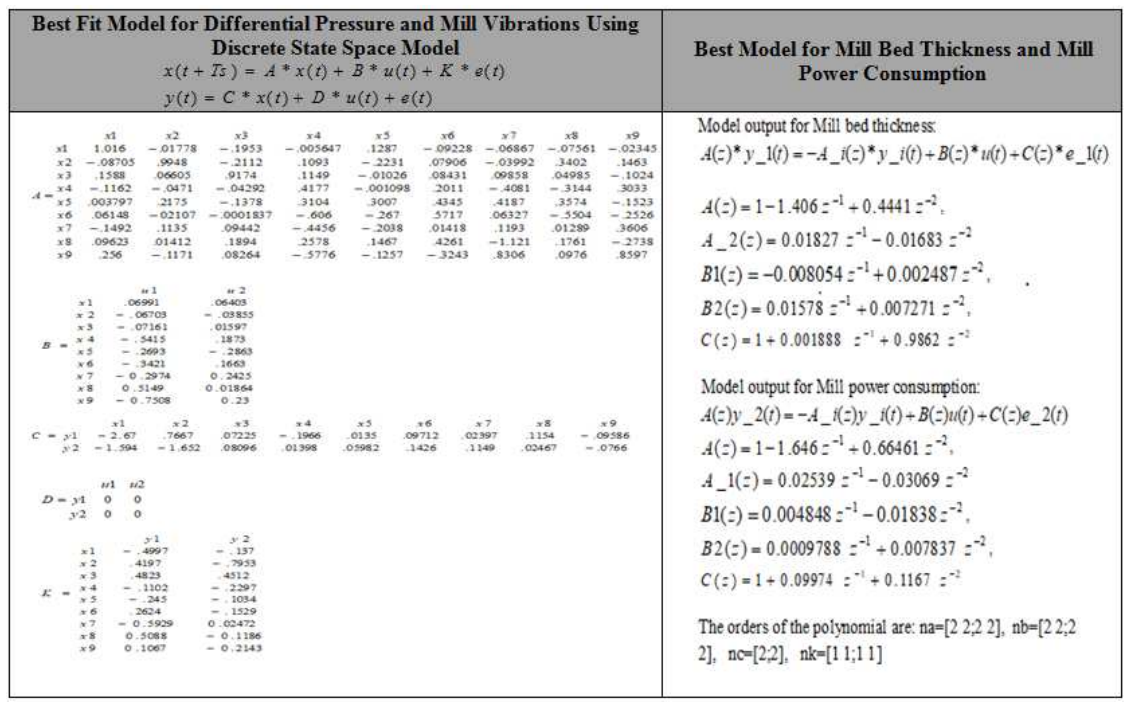

\section{CONCLUSIONS AND FUTURE SCOPE}

Simulation results show satisfactory prediction capabilities for VRM modelling. After comparing the results state space and Nonlinear ARX models gives better fitted models when compared with their counterparts. This work can be extended in future by applying methods like neural network time series, fuzzy logic, spectral and correlation.

\section{REFERENCES}

1. Yan-yan NING, Guang ZHU, Yuan Ming-zhe and Zhuo WANG. 2011. Design of Intelligent control system for Vertical Roller Mill. IEEE $2^{\text {nd }}$ International conference on Intelligent control and Information processing. 315-318

2. Yewang SUN, Xiangbo ZE, Xuefeng YANG, Chaoyang ZHANG and Yiqi ZHOU. 2009. Analysis of Flow Field in Vertical Roller Mill Chamber Based on Turbulent Models. IEEE International Conference on Environmental Science and Information Application Technology. 637-640

3. Kourosh Danai. 2015. Signature-Based Time-Series Analysis for System Identification. IEEE Control systems magazine. 40-70

4. Arab AlSharif and Manohar Das. 2013. A Time-varying Transfer Function Model for Modeling the Charging Process of a Lithium-ion Battery. IEEE Energytech. 1-7 
5. Mitchell A. Thornton. 2015. Simulation and Implication Using a Transfer Function Model for Switching Logic. IEEE Transactions on computers, 64. 3580-3590

6. Vijaya Bhaskar B. and Jayalalitha S. 2017. Process control for cement grinding in vertical roller mill (VRM) a review. ARPN Journal of Engineering and Applied Sciences. 5758-5765

7. Rafael Rui, Tohid Ardeshiri, Henri Nurminen, Alexandre Bazanella and Fredrik Gustafsson. 2017. State Estimation for a Class of Piecewise Affine State-Space Models. IEEE Signal processing letters. 61-64

8. Jose Luis Pitarch, Antonio Sala and Carlos Vicente Arino. 2014. Closed-Form Estimates of the Domain of Attraction for Nonlinear Systems via Fuzzy- Polynomial Models. IEEE Transactions on cybernetics. 526-538

9. Tara Baldacchino, Sean R Anderson, Visakan Kadirkamanathan. 2012. Structure detection and parameter estimation for NARX models in a unified EM framework. Automatica. 857-865

10. Brown M. D., Lightbody G., and Irwin G. W. 1997. Nonlinear internal model control using local model networks. IEE Process Control Theory. pp. 505-514

11. Chow Yin Lai, Cheng Xiang and Tong Heng Lee. 2011. Data-Based Identification and Control of Nonlinear Systems via Piecewise Affine Approximation. IEEE Transactions on neural networks. 2189-2199

12. Adrian Wills, Thomas B. Schon, Lennart Ljung and Brett Ninness. 2012. Identification of Hammerstein-Wiener Models. Automatica. 1-7

13. Michaël Boulvin, Alain Vande Wouwer, Renato Lepore, Christine Renotte, and Marcel Remy. 2003. Modeling and Control of Cement Grinding Processes. IEEE Transactions on control systems technology. 715-724

14. Boulvin M. Renotte, Vande Wouwer A., Remy M., Tarasiewiczt S., and Cesar P. 1997. Modeling, simulation and evaluation of control loops for a cement grinding process. European Control Conference (ECC). 1334-1339 
